\title{
Research and analysis of the issue of flight stability of an unmanned aerial vehicle
}

\author{
LUCJAN SETLAK, RAFAŁ KOWALIK \\ Aviation Division, Department of Avionics and Control Systems \\ Polish Air Force University \\ Deblin 08-521, ul. Dywizjonu 303 No. 35 \\ POLAND
}

\begin{abstract}
The main goal of this work was to develop a mathematical model in the field of UAV object flight dynamics, taking into account the stabilization of its location in space in such a way that the balancing state of the flying object can be ensured despite both atmospheric and equipment disturbances. Mathematical model of quadcopter dynamics developed using the Newton-Euler method, which describes the exact relationship between all variables, significant in the context of the movement of the object in the coordinate system. For this purpose, it was necessary to derive linear equations regarding state spaces intended for the design of the controller and its further evolution. It should be noted that the issues related to the stability of the quadrotor flight in terms of making turns and wear during operation (explosion) of its propellers are due to the aerodynamic force and gyroscopic effect during take-off and landing. In addition, vibrations resulting from the influence of atmospheric turbulence are an additional disturbance indirectly affecting the stability of the UAV object. The created mathematical model of the quadcopter was developed in the Matlab/Simulink programming environment, and the obtained test results at the stage of simulation tests were presented in the form of tilts in the motion of the considered quadrotor object. In the final stage of this article, based on the UAV object research (mathematical model, simulations, tests) and analysis of the results obtained, practical conclusions were formulated.
\end{abstract}

Key-Words: - Testing and analyzing, stability of the object, unmanned aerial vehicles

Received: August 19, 2019. Revised: January 4, 2020. Accepted: January 21, 2020.

Published: January 31, 2020.

\section{Introduction}

Currently, modern unmanned aerial vehicles (UAVs) are increasingly used in the use of them for long-term flights, characterized by the requirement both in terms of the need to take more fuel, as well as in the context of equipping drones with heavier but more efficient sources power supply in the form of accumulator batteries [1], [2], [3].

This situation obliges designers to design a larger platform size or to implement a solution to significantly reduce the lifting capacity of the UAV object.

In addition, constantly rising fuel costs lead to increased competition among flying object engineers in the aspect of savings in terms of fuel consumption during the flight.

One of the key solutions is the search for ways to reduce energy demand required for long-term flight of the flying object, mainly by reducing aerodynamic drag.

In addition, it should be noted that an interesting, but still not fully explored area are, among others unconventional aerodynamic constructions.
They mainly include constructions with reduced or no tail section, i.e. flying wing systems or a newer concept, i.e. the BWD (blended wing body).

It is estimated that their use can provide savings of up to about $25 \%$ less in-flight resistance compared to the latest conventional flying apparatuses [4], [5].

The flying wing is an aerodynamic system that does not have a clear division between the fuselage and the wings, unlike the flying wing system, which does not have a distinctive fuselage. In addition, the BWD design usually does not have a tail section.

The potential advantages of this type of solution are wings with a high lift coefficient, i.e. supporting fuselage, which means that the structure as a whole produces lifting force reducing the size and thus the resistance generated by the wings as a separate part of the object [6], [7], [8].

The blended wing is characterized by much greater aerodynamic excellence compared to a conventional aircraft, ensuring greater fuel savings.

This type of construction, despite the potential profits and possible applications, also has properties 
that require the use of unconventional technical solutions.

It should be noted that in conventional constructions the center of gravity is slightly in front of the pressure center, which causes the aircraft to tend to fly downwards.

Horizontal stabilizers placed in the tail part of the aircraft create an anti-motion moment, which allows a steady flight of the aircraft (flying in a straight line, without changing the height of the flight without the intervention of the pilot).

It should be noted that in the case of a blended wing, the moment action arm counteracting unbalancing moments is short, the issue of the position of the center of mass of the aircraft begins to be a significant problem [9], [10].

In addition, the lack of horizontal stabilizers means that to ensure an adequate reserve of longitudinal stability it is necessary to use an electronic control system FBW (fly-by-wire).

\section{Object reference systems}

Complete aircraft characteristics are obtained by combining three reference systems associated with the aircraft and an inertial reference system related to the UAV object [11], [12], [13].

It is assumed that the total movement of the UAV object is a combination of the body center of gravity motion and rotational motion of the body as a rigid body.

The center of gravity movement results from the position of the axis in the aerodynamic reference system over time.

The total movement of the body as a rigid body therefore arises from the position of the axis in the reference system associated with the aircraft, relative to the axis in the aerodynamic reference system [14], [15], [16].

In the context of the needs of further considerations in the field of object stability, an aerodynamic reference system is used.

\subsection{Inertial reference system (Earth-related)}

The inertial reference system is the only system in which Newton's laws of dynamics are applied. It is assumed that the Earth is flat and not rotating (Fig. 1).

Given the typical length and the duration of local UAV flights, and the fact that the angular velocities of the aircraft are much higher compared to the angular velocity of the Earth, the assumption of a flat plane is justified [17], [18].
The horizontal plane in this reference system coincides with the surface of the Earth, and the origin of the reference system is at the most suitable point in the case under consideration, e.g. a runway.

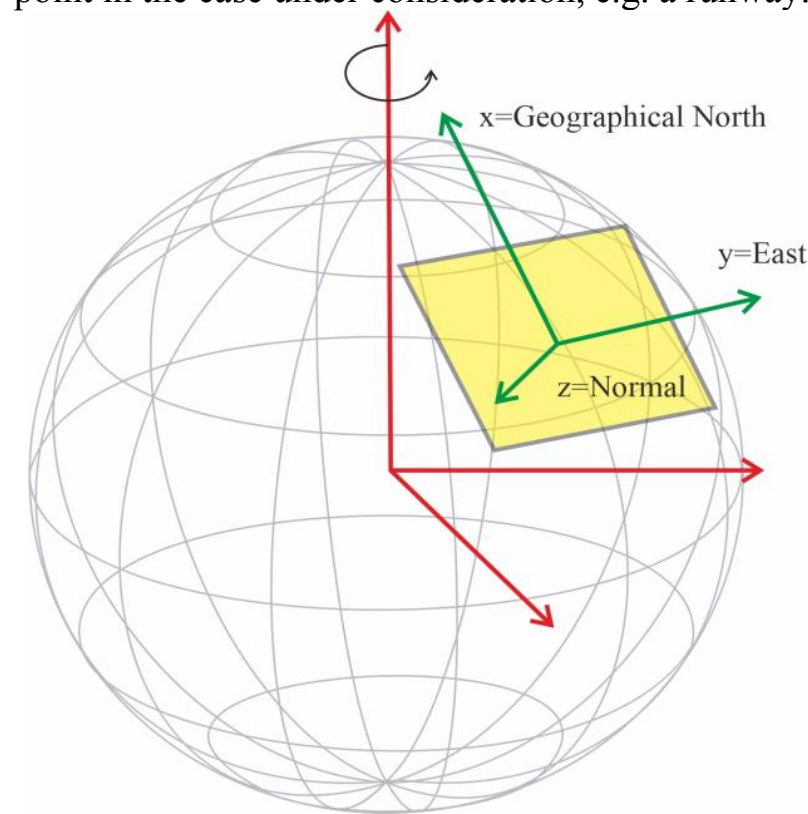

Fig. 1 Inertial reference system

\subsection{The reference system associated with the body}

The reference system is associated with the body, with its origin in its center of gravity (Fig. 2). It is assumed that gravity is the same at every point, hence the center of gravity coincides with the center of body mass [19], [20], [21].

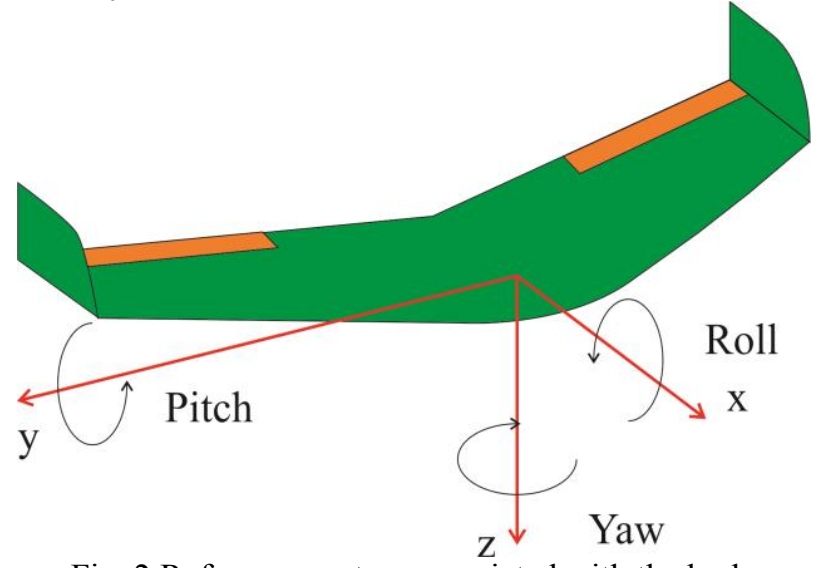

Fig. 2 Reference system associated with the body

\subsection{The reference system associated with the flow}

The reference system is similar to the body related reference system in axis directions, except for the $X$ axis, which is always parallel to the flow velocity vector [22], [23], [24]. 
The $Z$ axis is in the plane of symmetry of the aircraft, while the $Y$ axis perpendicular to the other two axes is directed towards the right wing (Fig. 3).

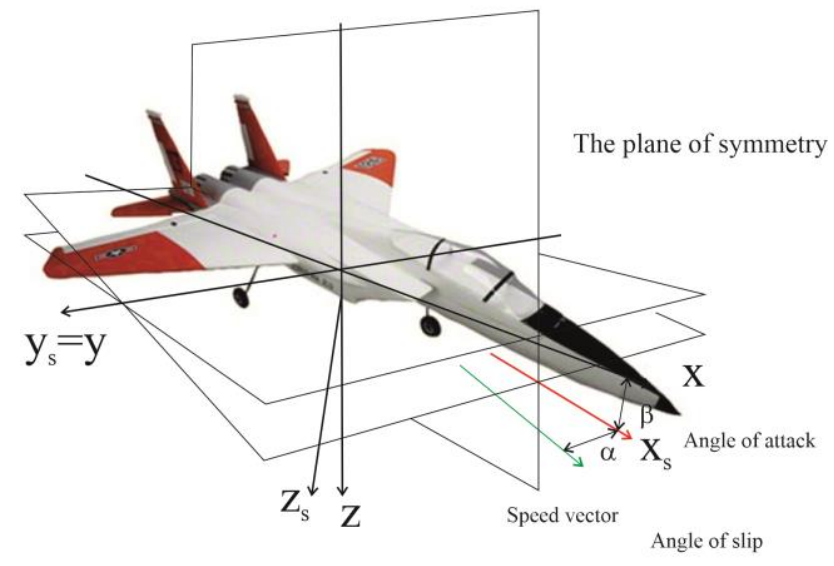

Fig. 3 Aerodynamic reference system

\section{Control surfaces}

Control instruments are aerodynamic devices used to rotate the aircraft around its selected axis. The tested UAV is equipped with 6 steering gears, respectively: $\left(\delta_{1}, \delta_{2}, \delta_{3}, \delta_{4}, \delta_{5}, \delta_{6}\right)$.

When modeling a classic airplane, a standard rudder system is used, namely: elevator $\left(\delta_{E}\right)$, rudder $\left(\delta_{R}\right)$ and ailerons $\left(\delta_{A}\right)$ [25], [26], [27].

To facilitate the creation of the BSP model, it is necessary to determine the relationship between the deflections of individual control devices in the UAV $\left(\delta_{1}, \delta_{2}, \ldots, \delta_{6}\right)$ and the forces, as in the classic model $\left(\delta_{E}, \delta_{R}, \delta_{A}\right)$.

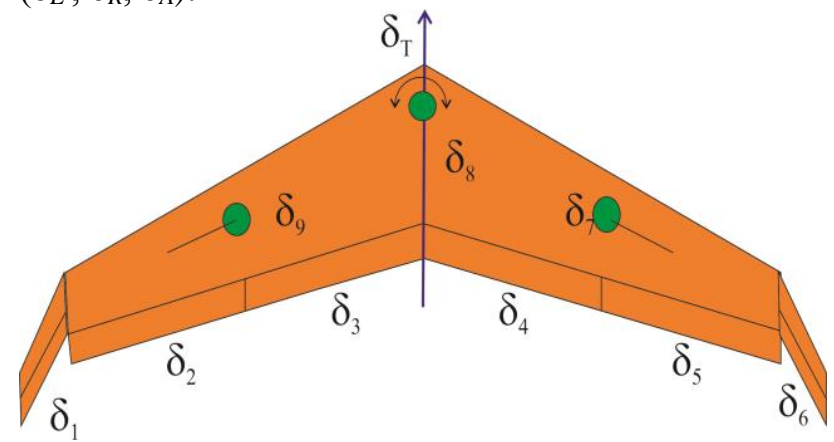

Fig. 4 Control surfaces arrangement

Control surface deflection is determined in radians, where a positive deflection causes a negative moment.

Surfaces $\delta_{1}, \delta_{2}, \ldots, \delta_{6}$, which act as rudder, work synchronously (Fig. 4). Both parts of the wing have identical sets of aerodynamic devices [28], [29].
For control purposes it is desirable that the control surfaces on one part of the wing swing simultaneously.

Therefore, according to the conditions described above, the following relationships (1), (2) and (3) can be established [30], [31]:

$$
\begin{aligned}
& \delta_{4}=\delta_{5} \\
& \delta_{1}=\delta_{6} \\
& \delta_{2}=\delta_{3}
\end{aligned}
$$

In order to link the arrangement of devices in UAV with devices in the classic control system, apparent, replacement control devices (4), (5) and (6) are created:

$$
\begin{gathered}
\delta_{E}=\left(\delta_{2}+\delta_{3}+\delta_{4}+\delta_{5}\right) / 4 \\
\delta_{A}=\left(-\delta_{2}-\delta_{3}+\delta_{4}+\delta_{5}\right) / 4 \\
\delta_{R}=\left(\delta_{1}+\delta_{6}\right) / 2
\end{gathered}
$$

By combining the above equations, the relationship between real and substitute control devices can be represented in the form of a matrix (7):

$$
\delta^{V}=\left[T^{V R}\right] \delta^{R}
$$

where: $\mathrm{V}$ and $\mathrm{R}$ in superscript represent substitute and real control devices respectively.

Considering the above, $\delta^{\mathrm{V}}$ and $\delta^{\mathrm{R}}$ can be considered as (8), (9):

$$
\begin{aligned}
\delta^{V} & =\left[\begin{array}{lllllllll}
\delta_{R} & \delta_{E} & \delta_{A} & \delta_{T} & \delta_{T} & \delta_{S} & 0 & 0 & 0
\end{array}\right]^{T} \\
\delta^{R} & =\left[\begin{array}{lllllllll}
\delta_{1} & \delta_{2} & \delta_{3} & \delta_{4} & \delta_{5} & \delta_{6} & \delta_{7} & \delta_{8} & \delta_{9}
\end{array}\right]^{T}
\end{aligned}
$$

The matrix transforming the deflections of real control devices into the deflections of replacement control devices is as follows (10) [32], [33], [34]:

$$
T^{V R}=\left[\begin{array}{ccccccccc}
\frac{1}{2} & 0 & 0 & 0 & 0 & \frac{1}{2} & 0 & 0 & 0 \\
0 & \frac{1}{4} & \frac{1}{4} & \frac{1}{4} & \frac{1}{4} & 0 & 0 & 0 & 0 \\
0 & -\frac{1}{4} & -\frac{1}{4} & \frac{1}{4} & \frac{1}{4} & 0 & 0 & 0 & 0 \\
0 & 0 & 0 & 0 & 0 & 0 & 1 & 0 & 0 \\
0 & 0 & 0 & 0 & 0 & 0 & 0 & 1 & 0 \\
0 & 0 & 0 & 0 & 0 & 0 & 0 & 0 & 1 \\
1 & 0 & 0 & 0 & 0 & -1 & 0 & 0 & 0 \\
0 & 1 & -1 & 0 & 0 & 0 & 0 & 0 & 0 \\
0 & 0 & 0 & 1 & -1 & 0 & 0 & 0 & 0
\end{array}\right]
$$

Control devices such as drive and landing gear are unambiguous mappings (one-to-one mapping of data). 
The virtual actuator $\delta_{\mathrm{S}}$ on the ground is controlled by signals coming from the rudder, while it is inactive during flight.

The relationship between actual control surfaces and specific replacement control devices can be definitively recorded as (11):

$$
\delta^{R}=\left[T^{V R}\right]^{-1} \delta^{V}
$$

It should be noted that with the help of the relationships established above, the modeling process is greatly facilitated by the possibility of using the definition of classic control devices (ailerons, rudder, elevator, propulsion).

After obtaining the appropriate model, defined with the help of replacement devices, it can easily be converted to the model determined by real devices using equation (11).

\subsection{Parameters of stability and control of the UAV object}

The change of force or moment caused by the change of normalized motion variables or control surface deflections are described by dimensionless quantities.

These types of parameters can be calculated from the first principle of dynamics, numerical methods for calculating fluid dynamics, wind tunnel measurements, in-flight data, and during identification of control systems [35], [36].

In addition, the above parameters allow comparison of aircraft of different sizes.

By expressing dimensionless stability and control parameters in an aerodynamic system, the following equations (12), (13) and (14) are obtained:

$$
C_{D}=C_{D_{0}}+\frac{C_{L}^{2}}{\pi A e}
$$

$$
\begin{aligned}
& {\left[\begin{array}{l}
C_{y} \\
C_{L}
\end{array}\right] } \\
= & {\left[\begin{array}{c}
0 \\
C_{L_{0}}
\end{array}\right] } \\
+ & {\left[\begin{array}{ccccc}
0 & C_{\Upsilon_{\beta}} & \frac{b}{2 V_{a}} C_{\Upsilon_{p}} & 0 & \frac{b}{2 V_{a}} C_{\Upsilon_{r}} \\
C_{L_{\alpha}} & 0 & 0 & \frac{\bar{c}}{\overline{2 V_{a}}} C_{\mathrm{L}_{q}} & 0
\end{array}\right]\left[\begin{array}{l}
\alpha \\
\beta \\
p \\
q \\
r
\end{array}\right] } \\
+ & +\left[\begin{array}{cccc}
C_{\Upsilon_{\delta_{A}}} & 0 & C_{\Upsilon_{\delta_{R}}} \\
0 & C_{\mathrm{L}_{\delta_{E}}} & 0
\end{array}\right]\left[\begin{array}{c}
\delta_{A} \\
\delta_{E} \\
\delta_{R}
\end{array}\right]
\end{aligned}
$$

$$
\begin{aligned}
& {\left[\begin{array}{c}
C_{l} \\
C_{m} \\
C_{n}
\end{array}\right] } \\
&= {\left[\begin{array}{c}
0 \\
C_{m_{0}} \\
0
\end{array}\right] } \\
&+\left[\begin{array}{ccccc}
0 & C_{l_{\beta}} & \frac{b}{2 \bar{V}_{a}} C_{\mathrm{l}_{p}} & 0 & \frac{b}{2 \bar{V}_{a}} C_{\mathrm{l}_{r}} \\
C_{m_{\alpha}} & 0 & 0 & \frac{\bar{c}}{2 \bar{V}_{a}} C_{\mathrm{m}_{q}} & 0 \\
0 & C_{n_{\beta}} & \frac{b}{2 \bar{V}_{a}} C_{\mathrm{n}_{p}} & 0 & \frac{b}{2 \bar{V}_{a}} C_{\mathrm{n}_{r}}
\end{array}\right]\left[\begin{array}{l}
\alpha \\
\beta \\
p \\
q \\
r
\end{array}\right] \\
&+\left[\begin{array}{ccc}
C_{l_{\delta_{A}}} & 0 & C_{l_{\delta_{R}}} \\
0 & C_{m_{\delta_{E}}} & 0 \\
C_{n_{\delta_{A}}} & 0 & C_{n_{\delta_{R}}}
\end{array}\right]+\left[\begin{array}{l}
\delta_{A} \\
\delta_{E} \\
\delta_{R}
\end{array}\right]
\end{aligned}
$$

For the purpose of this work, the movement of whirling wind simulation program of the AVL company was used, which created its own standard in the field of AST (Advanced Simulation Technology) simulation process technology. This is to determine stability and control parameters.

In equation (12), $A$ - defines airframe elongation, $C_{D_{0}}$ - coefficient, and $e$ - defines Oswald's efficiency factor.

However, in equations (13) and (14), $C_{L_{0}}$ - is the static lift coefficient, while $C_{m_{0}}$ - is the static moment coefficient.

Shape conditions can be written in the following form (15) [37]:

$$
C_{\lambda_{\epsilon}}=\frac{\partial C_{\lambda}}{\partial \epsilon^{\prime}}
$$

where: $\epsilon$ is equal to (16):

$$
\epsilon^{\prime}=n \epsilon
$$

Shape conditions recorded in the form of equation (15) represent dimensionless stability and control parameters with $n$ as the normalizing factor $\epsilon$.

For the angular velocity of inclination, the normalizing factor is $\frac{\bar{c}}{2 \bar{V}_{a}}$, and for the tilting and deflection velocity $\frac{b}{2 \bar{V}_{a}}$.

Thus, any change in $\epsilon^{\prime}$ causes a change in $C_{\lambda}$ and is recorded as $C_{\lambda_{\epsilon}}$. In turn, strength and the moment is symbolized by $\lambda$, while the normalized kinematic state - by $\epsilon^{\prime}$ symbol.

In the presented model, the first degree stability parameters were omitted. Only first-level parameters $C_{L_{\alpha}}$ and $C_{m_{\alpha}}$ quantifying effects such as, among others, flow (downwash lag). For the 
blended wing, flow is negligible due to the lack of the tail section of the airframe, hence it is assumed that the additional mass is negligibly small for modeling calculations.

Additionally, it is assumed that the stability and control parameters are not a function of the rotational dynamics of the rigid body, but rather the parameters of this dynamics.

It should be noted that this type of assumption is true for the operating aircraft in a narrow range of the angles of attack and significantly simplifies the analysis of the control system.

\subsection{The forces of gravity}

Rotational dynamics equations require coordination of the gravity vector with the axes of the aerodynamic system. For this purpose, a rotation matrix is used to coordinate the gravity vector $(G)$, having a fixed position in the inertial space, with the axes of the aerodynamic system (W).

The moment of gravity is zero, because the vector of this force passes through the center of gravity, which can be written in the form of the following equation (17):

$$
\left[\begin{array}{c}
X_{W}^{G} \\
\Upsilon_{W}^{G} \\
Z_{W}^{G}
\end{array}\right]=\left[D C M^{W \mathrm{I}}\right]\left[\begin{array}{c}
0 \\
0 \\
m g
\end{array}\right]
$$

where: $g$ - is gravitational acceleration, $m$ - mass of the aircraft, and $D C M^{W \mathrm{I}}$ - is the transforming matrix of rotation.

\section{Sample simulation results in the AVL program}

As mentioned earlier, AVL is software that uses modeling methods using the network of whirls.

The drawings below (Fig. 5-8) illustrate how stability and control parameters are discussed in part change with a linear change in the center of mass position within the range from a static position to a statically non-static position.

In the figures below, a value of $0 \%$ means a change in the center of mass position, corresponding to the case when the aircraft is statically stable, and $100 \%$ is the case in which the change occurs when the center of mass is in the extreme rear statically unstable position.

The data presented in this part (chapter 4) correspond and are valid for the model used by one of the scientific circles of the Polish Air Force University.
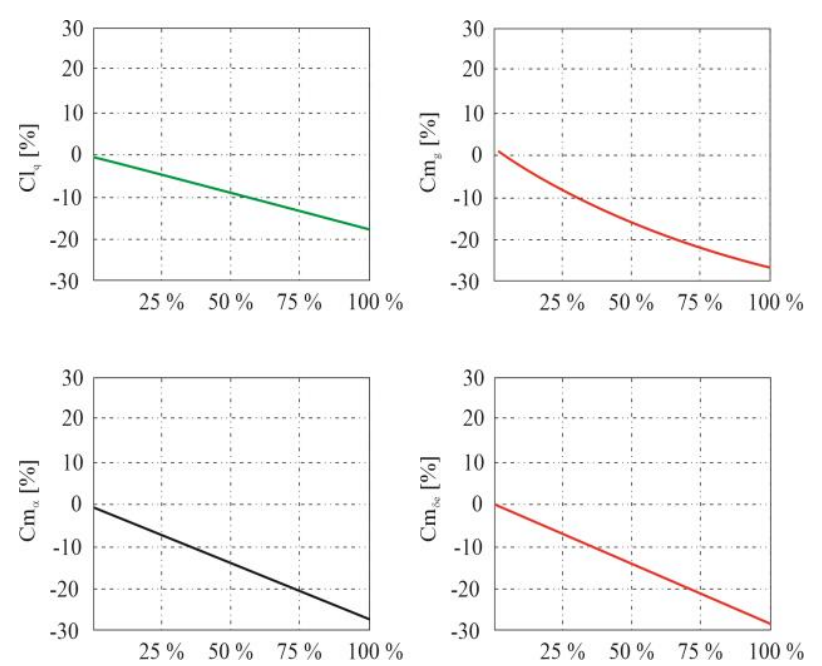

Fig. 5 Percentage change in parameters $C_{L_{q}}, C_{m_{q}}, C_{m_{\alpha}}$,

$C_{m_{\delta_{E}}}$ due to the change in the center of mass position

In the figure above (Fig. 5), the parameters $C_{L_{q}}$, $C_{m_{\alpha}}$ i $C_{m_{\delta_{E}}}$ change linearly with the position of the center of mass, with the $C_{m_{q}}$ parameter showing a quadratic relationship with the tested position of the center of mass.

In addition, the parameters $C_{m_{\delta_{E}}}$ and $C_{m_{q}}$, depending on the moment arm $l_{T}$, show an approx. $25 \%$ change in the function of the center of mass position change, which means that the aerodynamic center of the replacement horizontal stabilizer must be in front of the edge of the proper wing.
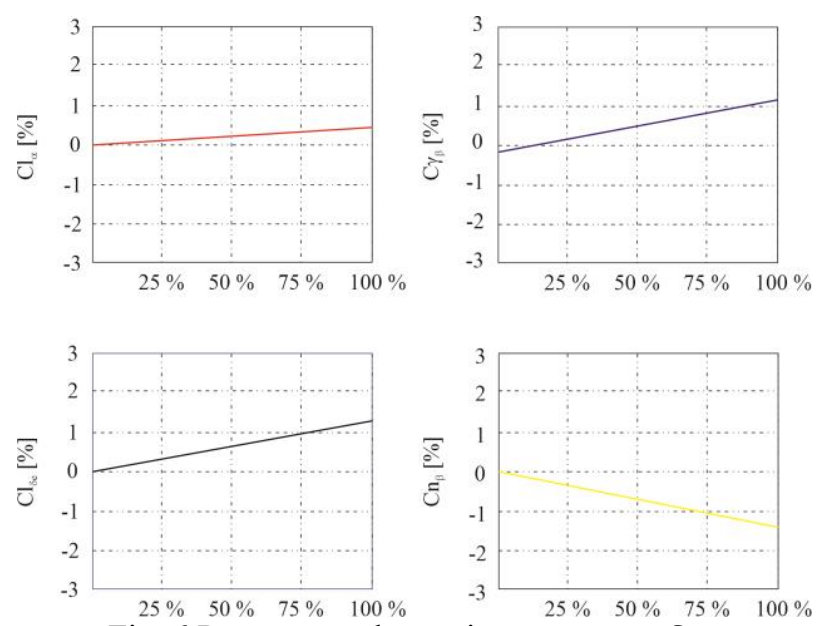

Fig. 6 Percentage change in parameters $C_{L_{\alpha}}$,

$C_{\Upsilon_{\beta}}, C_{L_{\delta_{E}}}, C_{\mathrm{n}_{\beta}}$ due to changes in the center of mass position

The above figure (Fig. 6) confirms the reasoning carried out in [38] showing that the parameters $C_{L_{\alpha}}$, $C_{\Upsilon_{\beta}}$ and $C_{L_{\delta_{E}}}$ basically remain unchanged in relation to the position of the center of mass.

In addition, the parameter $C_{n_{\beta}}$ shows an approx. $10 \%$ change, therefore for the purposes of the 
control system analysis it is assumed that it remains constant with the change of the center of mass position.

In turn, the next figure (Fig. 7) shows that the parameters $C_{\Upsilon_{\delta_{R}}}, C_{\mathrm{l}_{\delta_{R}}}$ and $C_{\mathrm{l}_{\delta_{A}}}$ remain constant with a change in the position of the center of mass, because they change by $4 \%$, confirming the arguments included in [38].

The parameter $C_{\Upsilon_{\delta_{A}}}$ shows a change of less than $15 \%$, therefore, similarly to $C_{n_{\beta}}$ is recognized for the purposes of the control system analysis, it remains constant when the center of mass position changes.
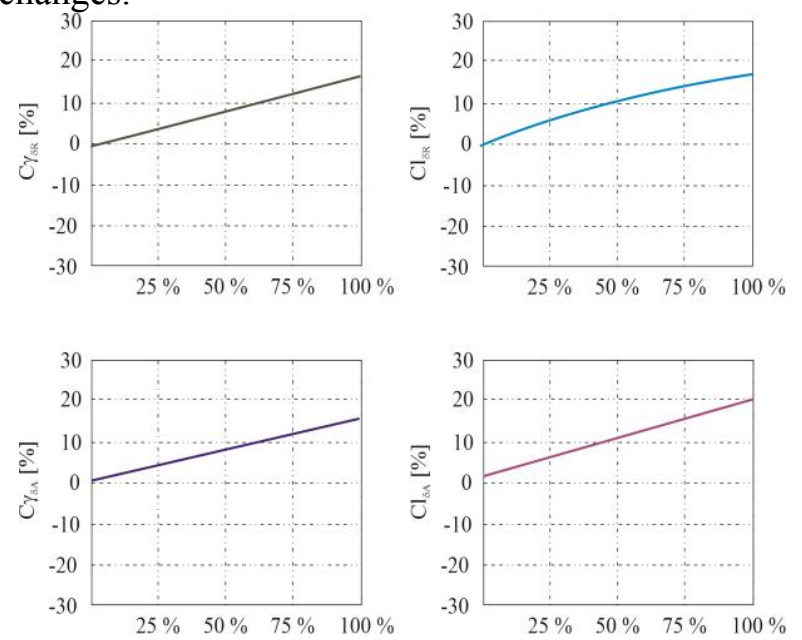

Fig. 7 Percentage change in parameters $C_{\Upsilon_{\delta_{R}}}$

$C_{\mathrm{l}_{\delta_{R}}}, C_{\Upsilon_{\delta_{A}}}, C_{\mathrm{l}_{\delta_{A}}}$ due to changes in the center of mass position

Data for modeling the object presented in the next figure (Fig. 8) show compliance with the arguments included in [38], because the parameters $C_{\mathrm{n}_{\delta_{R}}}, C_{\Upsilon_{r}}, C_{l_{r}}$ and $C_{l_{p}}$, change by less than $10 \%$, which means that for the purposes of analysis of the control system they are considered constant at the changing center of mass.
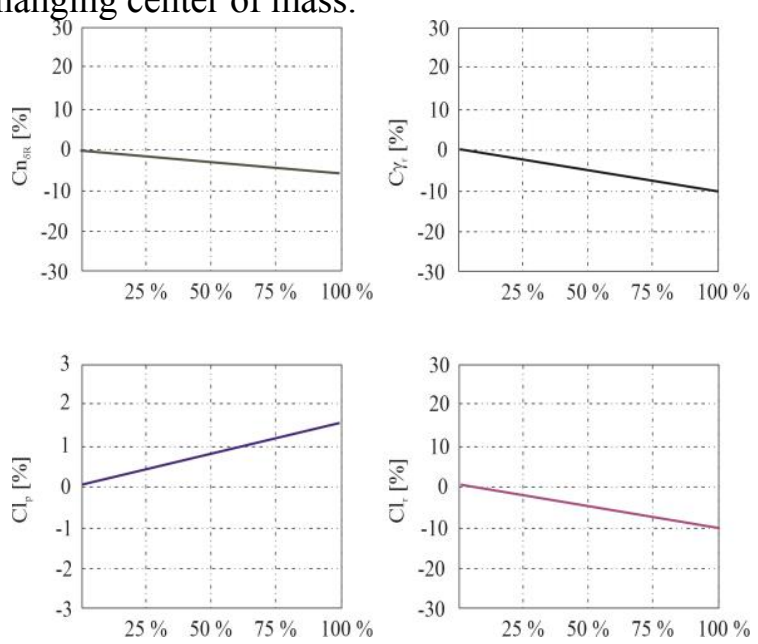

Fig. 8 Percentage change in parameters $C_{\mathrm{n}_{\delta_{R}}}, C_{\Upsilon_{r}}, C_{\mathrm{l}_{p}}, C_{\mathrm{l}_{r}}$ due to changes in the center of mass position

\section{Conclusions}

A detailed analysis of key stability and potential control parameters of the object carried out in Chapter 4 and the results of their simulation suggest that the most important parameters from the point of view of lateral motion dynamics can be considered to be independent of changes in the center of mass position with a high degree of certainty.

It is clearly shown that with the change of position of the center of mass, the lateral stability remains at an acceptable level, simultaneously with the significantly changing longitudinal stability.

This causes that the problem of stability changes is the same as for a conventional aerodynamic system, which also allows the use of conventional solutions in the scope of tilt and deviation control, when designing the control system.

It should be noted that all stability and control parameters occur as linear or to significant insights (Figs. 5-8) assumption (through the possible use of quadratic center of gravity position functions for possible verification of simulation software) for conventional lateral control system using complete dynamics of variable stability aircraft.

In addition, the analysis of the most important results for longitudinal stability leads to important conclusions and justifies longitudinal stability as the most important problem when considering the overall stability of an aircraft.

\section{References:}

[1] J. D. Becker, "Modeling and Control of a Quadrotor with Dynamic Inertia," PhD thesis, 2013.

[2] R. Whittle, Predator: The Secret Origins of the Drone Revolution, Macmillan, 2014.

[3] L. Setlak, R. Kowalik, "Studies of 4-rotor unmanned aerial vehicle UAV in the field of control system," 22nd International Conference on Circuits, Systems, Communications and Computers, CSCC 2018, MATEC Web of Conferences, Vol. 210, 2018.

[4] W. Xiao "Research on the control technology of lateral coupling for hypersonic vehicle, Nanjing University of Aeronautics and Astronautics, Nanjing, (2014).

[5] J. M. Pflimlin, P. Soueres, T. Hamel, "Position control of a ducted fan VTOL UAV in crosswind," Int. J. Control 80(5): 666-683, (2007).

[6] K. T. Oner, E. Cetinsoy, M. Unel, M.F. Aksit, I. Kandemir, and K. Gulez, "Dynamic model and control of a new quadrotor unmanned aerial vehicle with tilt-wing mechanism," 
World Academy of Science, Engineering and Technology, Vol. 45, 2008.

[7] S. Datta, "Multicopters with variable flight characteristics," July 2015.

[8] L.M. Argentim, W.C. Rezende, P.E. Santos, and R. A. Aguiar, "PID, LQR and LQR-PID on a quadcopter platform," in Informatics, Electronics Vision (ICIEV), 2013 International Conference, 2013, pp. 1-6.

[9] Y.L. $\mathrm{Xu}$, "Crash source Investigation on the safety of take-off and landing," DB/OL, Xinhua (2011-2015).

[10] Y. G. Tan "The dynamic analysis of a controllable under actuated robot," Wuhan University of Technology, Wuhan (2013).

[11] N. Michael, D. Mellinger, Q. Lindsey, and V. Kumar, "The grasp multiple micro UAV testbed," IEEE Robotics Automation Magazine, Vol. 17, September 2010, pp. 56-65.

[12] D. Mellinger, N. Michael, M. Shomin, and V. Kumar, "Recent advances in quadrotor capabilities," in Robotics and Automation (ICRA), 2011 IEEE International Conference, May 2011, pp. 2964-2965.

[13] J.P. Dyhr, N. J. Cowan, D.J. Colmenares, K.A. Morgansen, and T.L. Daniel, "Auto stabilizing airframe articulation: Animal inspired air vehicle control," in Decision and Control (CDC), 2012 IEEE 51st Annual Conference, IEEE, 2012, pp. 3715-3720.

[14] L. Setlak, R. Kowalik, "The dynamics of group flights of an unmanned aerial vehicle," WSEAS Transactions on Applied and Theoretical Mechanics, Volume 14, 2019, pp. 129-139.

[15] M. M. "Dong Design and dynamic analysis of a helicopter landing gear parameters," Nanjing University of Aeronautics and Astronautics, Nanjing (2010).

[16] D. W. Yoo, H. D. Oh, D. Y. Won, and M. J. Tahk, "Dynamic modeling and control system design for trirotor UAV," in Systems and Control in Aeronautics and Astronautics (ISSCAA), 2010 3rd International Symposium, June 2010, pp. 762-767.

[17] S. Sadr, S. A. A. Moosavian, and P. Zarafshan, "Dynamics modeling and control of a quadrotor with swing load," Journal of Robotics, 2014.

[18] J. Villagomez, M. Vargas, and F. Rubio, "Backstepping and sliding-mode technique sapplied to an under actuated camera onboard a rotorcraft mav," in 3rd Workshop on Visual Control of Mobile Robots, Vicomor, 2014, pp. 1-7.

[19] F. Senkul, and E. Altug, "Modeling and control of a novel tilt - roll rotor quadrotor UAV," in
Unmanned Aircraft Systems (ICUAS), 2013 International Conference, May 2013, pp. 10711076.

[20] E. Jimenez-Cano, J. Martin, G. Heredia, A. Ollero, and R. Cano, "Control of an aerial robot with multi-link arm for assembly tasks," in Robotics and Automation (ICRA), 2013 IEEE International Conference, May 2013, pp. 49164921.

[21] D. Mellinger and V. Kumar, "Minimum snap trajectory generation and control for quadrotors," in Robotics and Automation (ICRA), 2011 IEEE International Conference, May 2011, pp. 2520-2525.

[22] A. Faust, I. Palunko, P. Cruz, R. Fierro, and L. Tapia, "Learning swing-free trajectories for UAVs with a suspended load," in Robotics and Automation (ICRA), 2013 IEEE International Conference, May 2013, pp. 4902-4909.

[23] L. Setlak, R. Kowalik, and S. Bodzon, "The study of air flows for an electric motor with a nozzle for an unmanned flying platform," WSEAS Transactions on Fluid Mechanics, Vol. 14, 2019, pp. 21-35.

[24] W.C. Zhang, S.C. Tan, P.Z. Gao, "Chaotic forecasting of natural circulation flow instabilities under rolling motion based on Lyapunov exponents, J. Acta Phys Sin 6(2013).

[25] M. Kumon, J. Katupitiya, I. Mizumoto, "Robust attitude control of vectored thrust aerial vehicles," In: 18th IFAC World Congress, Milano, Vol. 28(8), (2011), pp. 2607-2613.

[26] J. B. Dingwell, L. C. Marin "Kinematic variability and local dynamic stability of upper body motions when walking at different speeds," J. Biomech 39: (2006), pp. 444-452.

[27] S. Stephen, H. Rachel, H. Cheryl, "Quantifying stability using frequency domain data from wireless inertial measurement units, J Systemics Cybern Inform, 10(4): pp. 1-4, (2012).

[28] YQ Bai, H. Liu, ZY Shi et al., "Robust flight control of quadrotor unmanned air vehicles," J. Robot 34(5): pp. 519-524, (2012).

[29] L. Setlak, R. Kowalik, "Dynamics of the designed robotic manipulator in the CAD program," WSEAS Transactions on Applied and Theoretical Mechanics, Volume 14, 2019, pp. 66-74.

[30] S. Bouabdallah, A. Noth, and R. Siegwart, "PID vs. LQ control techniques applied to an indoor micro quadrotor," in Intelligent Robots and Systems, (IROS), Proceedings 2004 
IEEE/RSJ International Conference, Vol. 3, 2004, pp. 2451-2456.

[31] H.D. Nguyen, H.L. Vu, M. VOLKER, "Robust stability of differential-algebraic equations, J. Surv. Differ-Algebraic Equ (2013), pp. 63-95.

[32] Y. Sum, C.Q. Wu, "Stability analysis via the concept of Lyapunov exponents: a case study in optimal controlled biped standing. Int. J. Control (2012)85(12):1952-1966.

[33] L. Setlak, R. Kowalik, "Analysis, Mathematical Model and Simulation Tests of the Unmanned Aerial Vehicle Control System," AMCSE 2018, ITM Web of Conferences, Vol. 24, 2018.

[34] K. J. Latawiec, R. Stanisławski,; M. Łukaniszyn, W. Czuczwara, M. Rydel, "Fractional-Order Modeling of Electric Circuits: Modern Empiricism vs. Classical Science," Proceedings of the IEEE 2017 Progress in Applied Electrical Engineering (PAEE), IEEE 2017, pp. 1-4.

[35] Y.B. Li, S.X. Song, "Hovering control for quadrotor unmanned helicopter based on fuzzy self-tuning PID algorithm," Jnt. Control Eng., China (2013), 20(5): 910-914.

[36] D. Boddington, Radio-controlled Model Aircraft, Crowood Press, 2004.

[37] C. Yang, Q. Wu, "On stabilization of bipedal robots during disturbed standing using the concept of Lyapunov exponents. J. Robotica, (2006) 24:621-624.

[38] M. W. Mueller and R. D'Andrea, "Stability and control of a quadrocopter despite the complete loss of one, two, or three propellers," in Robotics and Automation (ICRA), 2014 IEEE International Conference, IEEE 2014, pp. 4552. 